\title{
Appraising the Utility of Internet-Mediated Communication for Qualitative Data Collection in Built Environment Research
}

\author{
Bankole Awuzie
}

Department of Built Environment, Centrl University of Technology, Westdene, Bloemfontein, 9300, South Africa

\begin{abstract}
Qualitative researchers within the built environment (BE) research community are confronted with data collection challenges. Such challenges have been attributed to the nature of the construction industry which has been described as fraught with high worker turnover rates and tight schedule of project stakeholders. These features diminish a researcher's chances of engaging in successful and in-expensive data collection exercises. A cursory look at studies reveals the fixation of qualitative researchers within the BE discipline on conventional qualitative data collection techniques whilst neglecting internet-mediated communication (IMC) techniques. This study contributes towards elucidating the usefulness of IMC techniques as a data collection approach for qualitative researchers in the built environment domain. An asynchronous online discussion forum (AODF) case study is deployed as an exemplar in this study. Advantages of ADOF highlighted in the reviewed include: its cost-effectiveness, automatic transcription, ability to reach discussants across various geographical contexts, etc. Also, its shortcomings, like the challenge of sustaining thread discussions were observed. In furtherance to this, it was discovered that the attainment of theoretical saturation with the AODF posed a challenge. It is expected that this study will contribute to the emerging discourse on the utility of such IMC techniques in the built environment domain, particularly with the evolving industry 4.0 agenda.
\end{abstract}

(C) 2018 The Authors. Published by Diamond Congress Ltd., Budapest University of Technology and Economics Peer-review under responsibility of the scientific committee of the Creative Construction Conference 2018.

Keywords: Asynchronous online discussion forums; Built environment; Internet-mediated Communication; Qualitative research

\section{Introduction}

Data collection continues to pose a challenge to researchers, especially qualitative researchers within the built environment (BE) research domain. Qualitative data has been eulogized for making significant contributions towards theory development as it facilitates the unbridled elicitation of the worldviews of prospective participants in research projects ${ }^{[6]}$. However, the process of collecting qualitative data can best be described as labourious. This is due to the presence of several challenges attributed to the temporary and fragmented nature of the industry, tight schedules experienced by project stakeholders within the construction environment, the geographically dispersed locations which the researcher must cover to reach a wider sample of potential interviewees or discussants ${ }^{[22]}$. This has been the lot of the qualitative researcher as issues of limited funding and timescales pose as significant obstacles to the seamless achievement of the research objective.

Yet, the conventional means of eliciting data through face-to-face interviews, focus group discussion as well as observation have remained predominant among qualitative researchers. As its central proposition, this study postulates that these approaches have negated the level of productivity experienced during data collection processes in qualitative research projects. It opines that overt dependence on the internet-mediated communication (IMC) techniques will lead to an increase in productivity levels without compromising on the quality and credibility of the findings emanating from the research project. 
The advent of the internet in the 80's and 90's has led to a transformational curve across various facets of contemporary living ${ }^{[25,27]}$. As such, significant improvements leading to efficiency and increased productivity have leveraged on this innovation. The educational sector remains a major beneficiary of the internet as the medium has been applied towards the development of pedagogical platforms for sharing knowledge between persons situated across geographically dispersed locations on a real-time, online basis without any time and distance barriers ${ }^{[21]}$. This has proven to be a cost-effective approach to pedagogy as many academic institutions have resorted to hosting their programmes through internet-/computer-mediated platforms and improving staff and student productivity ${ }^{[1]}$. As such, significant investments are continually being made by academic institutions at different levels to foster an internetdependent culture across the entire gamut of their institutional activities. Although the use of the IMC platforms appears to have been extensively deployed in improving teaching and learning processes in these institutions, it appears that it is yet, a new concept to qualitative researchers within the BE research space ${ }^{[16]}$. Partly, the apathy to the optimal utilization of IMC techniques in engendering effective and efficient data collection processes have resulted from the uncertainty surrounding its adoption and deployment ${ }^{[25]}$. Such uncertainty stems from issues like the acceptability of the findings resulting from the study, security of information shared over such platforms and willingness of participants to share such information across such platforms, the level of access to internet connectivity as well as ability to engage with the internet in certain contexts by certain categories of participants which may affect participation levels, etc. ${ }^{[16 \text {, }}$ 5].

But, ${ }^{[27]}$ Turney and Pocknee (2005) admit to the vast opportunity presented by the internet and internet-enabled platforms in supporting the researcher's ability to document data in a text format, providing conducive environments for participants to voice their opinions pertaining to a phenomenon. In furtherance to this, a review of existing literature reveals a paucity of literature detailing a procedural approach to the deployment of these IMC platforms by qualitative researchers, both within and outside the BE domain during data elicitation processes ${ }^{[11]}$. Perhaps, the abundance of such articles as is the case with the pedagogical facets will engender increased utilization of IMC platforms among researchers. This is a gap which this study seeks to bridge. ${ }^{[16]} \mathrm{Im}$ and Chee (2006) admit this when they bemoan the low level of awareness in the research community regarding the practical issues associated with the use of the online platforms for data elicitation. Accordingly, this study will provide an insight into the utility of such IMC platforms in the collection of qualitative data within the BE context, relying on an asynchronous online discussion forum (AODF) exemplar. Furthermore, it will highlight the advantages as well as shortcomings of the AODF experienced by the researcher during the study being presented and the steps taken to surmount these challenges.

To achieve its objective, this study will be structured thus: a review of relevant literature on the nature of online discussion forums; a reflection on the similarities between AODFs and conventional focus group discussion platforms; the nature of the construction industry and BE research; an exposé on the study within which the AODF was applied to collect data; a reflection on the drivers, merits, challenges and ethical considerations experienced during the deployment of the AODF in the study, and; a conclusion.

\section{Literature review}

\subsection{Online discussion forums}

The advent of the internet appears to have brought in its wake a paradigmatic shift in the way certain processes were executed ${ }^{[5,7,2]}$. They admit that the internet is a veritable platform for carrying out unbiased research and offers the researcher with an opportunity of experiencing a plethora of nuanced instances which may not have been possible with the conventional modes of data collection. Also, ${ }^{[25]}$ observe the burgeoning rise of the internet usage in the research community despite little reportage. They identify the utility of the internet in carrying out literature reviews, and the compilation of bibliographic databases among other aspects. The rise in the use of IMC platforms to facilitate borderless dissemination of knowledge as well as information sharing across multiple platforms evolved from this era. Online discussion forums are IMC platforms which have been extensively deployed towards knowledge creation/development and dissemination among different individuals across different locations. In elucidating the usefulness of online discussion forums, Gao et al (2013) ${ }^{[11]}$ catalogue a variety of uses to which such platforms can be 
put to. Examples of such uses include the development of online communities, enabling unbridled information and knowledge exchange, provision of a platform for collaborative problem-solving and critical and reflexive thinking.

Whereas online discussion forums have a long history of being applied in the pedagogical undertakings in academic institutions, its introduction to the research community can be described as relatively nascent ${ }^{[5,17,27,24]}$. However, they opine that its adoption within the research context has availed researchers with an opportunity to gain access to previously hard to reach groups. This was buttressed in a recent study ${ }^{[19]}$ wherein an online discussion forum was used gathering qualitative data from young people who have experienced breakups in romantic relationships and in another study, wherein online discussion forums where applied in understudying the perception of the youth on the burgeoning housing issues in their locality by ${ }^{[20]}$. Maestre et al. (2018) ${ }^{[18]}$ highlight the salient nature of online discussion forums in researching into stigmatized groups. Whilst it should be acknowledged that the participants in a multiplicity of BE research projects cannot be assigned the toga of a 'hard to reach' sample, it is often difficult to recruit probable participants. This difficulty is because of the different factors which have been mentioned previously in the preceding section. The presence of such challenges which are peculiar to BE context accentuates the need for the adoption and utilization of IMC platforms to gather data in an effective and efficient manner. Online discussion forums are classified according to two distinct categories, namely: synchronized and asynchronized online discussion forums ${ }^{[25]}$. Whereas the synchronized communication takes place online, real-time and instantaneously, the latter avails participants with an opportunity to provide answers at their convenience. Examples of the former include online chat-rooms and online interviewing platforms such as Skype and other types of voice over internet protocol (VOIP) platforms whereas online discussion forums/ boards can be classified as being asynchronous discussion platforms. Also, the asynchronous category allows for participants to join in on any discussion at their convenience without prompting, the synchronous category does not allow for this as it is instant ${ }^{[20]}$.

In this study, the intention is to provide a detailed account of the application of an AODF in the collection of qualitative data from a group of discussants who would ordinarily have been difficult to access based on their locations and time zones. In this wise, the AODF was utilized as a replacement of the conventional focus group discussion which appears to be prevalent in contemporary BE research.

\subsection{Asynchronous online Discussion Forums as Focus Groups}

As mentioned earlier, AODFs have been likened to conventional focus group discussion exercises albeit conducted on an IMC platform to engender wider participation ${ }^{[26]}$. Lim and Tan $(2001)^{[17]}$ compare the features of a focus group discussion forum and AODF and conclude that the AODF fared better. Aspects were the AODF was found to have proven advantageous in the comparative exercise include: convenience, reduced instances of social interference, reduction in group-think as individuals could air their views without coercion, opportunities for reflection by the discussants and adequate control was provided to facilitators. Furthermore, transcription was made easier given that discussants were expected to provide their opinions in textual format. Most of these advantages have been highlighted in recent studies such as ${ }^{[17]}$. Conversely, scholars have also identified the failings of the AODF in terms of the high incidence of discontinuous threads, the need for participants to possess the requisite computer literacy skills as well as the absence of non-verbal clues ${ }^{[17,12]}$.

Based on the foregoing, this study was carried out to buttress the salient benefits accruable from the deployment of AODFs in the collection of qualitative data by BE researchers. This is considered imperative in view of the increasingly new reputation of $\mathrm{BE}$ professionals as a 'hard to reach' sample owing to peculiarities associated with the industry.

\subsection{The Built Environment domain and its 'hard to reach' sample}

Role players within the $\mathrm{BE}$ context are often occupied on projects with tight time schedules. It is becoming increasingly difficult for researchers conducting studies within this context to reach them and elicit their views pertaining to problems beleaguering the industry. However, qualitative data remains critical to the development of context-dependent theory as well as its usefulness in gaining both in-depth understanding of a phenomenon as well as 
obtaining rich descriptions of happenstance in within a context ${ }^{[6,9]}$. The peculiar nature of the BE context makes the elicitation and subsequent use of qualitative data imperative.

Yet, the BE is a domain with a high turnover rate among role players given its task-based nature. Individuals exit the scene upon completion of assigned tasks. This transition and the fact that these role players are often located at several locations poses a significant challenge in scheduling interviews or focus group discussion sessions with them. Often, this is responsible for the incessant reportage of low response rates or participant apathy in qualitative research projects. As such, this study will highlight the utility of the AODF in overcoming identified challenges through a BEcentered study.

\subsection{The Study Context}

As part of a wider study seeking to investigate the implementation of socio-economic policies through procurement of oil and gas infrastructure in a developing country context, the researcher was confronted with the need to develop a framework for the proposed investigation. Having opted to situate this study within an organizational theory and systems thinking praxes, the researcher was confronted with the challenge of selecting an appropriate methodology for conducting an organizational diagnosis of the infrastructure procurement and delivery system. In the search for this methodology, it was agreed that such a methodology should not only enable but a diagnosis of the entire process of procuring and delivering infrastructure but also highlight in the extant relationships and responsibilities of the parties to the delivery process. Basically, such methodology should be able to describe in absolute terms the implementation framework for the procurement and delivery of oil and gas infrastructure in that country context as only such a representation would engender a succinct understanding of the entire process from a systemic perspective. Also, the implementation framework to be depicted in the proposed vignette will enable an accurate representation of the interorganizational relationship existing between the parties to the implementation process and the control and coordination points- items considered essential for systemic diagnosis of organizations.

Upon the probe of existing systems thinking, organizational theory and cybernetics theory literature, the researcher came across the term systems cybernetics and eventually, systems viability or viable systems theory as propounded in the seminal works of Sir Stafford Beer ${ }^{[4]}$. In the aftermath of a review of the viable systems approach to organizational viability and the conclusion of further inquest into its provenance in the organizational diagnosis context, the researcher arrived at the realization that the viable systems model (VSM)- a model premised on the principles of systems viability had only being applied towards diagnosing conventional organizations ${ }^{[14]}$. In this study, conventional organizations are considered as organizations that have a significant degree of permanence as when compared to organizations with a great degree of temporality. Organizations in the latter category are termed temporary organizations and, in the context, where such organizations consist of a plurality of organizations striving to deliver a certain product or service, they are termed temporary multi-organizations (TMO) ${ }^{[23]}$. The oil and gas infrastructure procurement and delivery system, hereinafter referred to as an Infrastructure delivery system (IDS) can be described as a TMO. This is because of the plethora of different organizations working in different capacities to deliver on the project objectives. Any diagnosis of the system will not be complete if it does not investigate the relationships between these organizations. As such, the researcher was unsure about the applicability of the VSM in the diagnosis of the IDS. There was an imminent need to reach out to system thinking as well as cybernetic experts to establish the model's applicability in this context.

Finding a community of experts, both in an individual and group capacity proved to be very arduous task. However, per chance, the researcher stumbled on two closed groups on LinkedIn housing communities of practice in the viable systems model and generic systems thinking. These groups will be referred to as VSA and ST groups respectively. Upon the identification of VSA and ST, the researcher proceeded to engage with the group administrators for admission onto these platforms.

The researcher obtained access to both platforms just a week apart with the VSA granting access first before the ST. In keeping with terms of disclosure, the researcher notified the two administrators of his intentions to utilize the platform to elicit data through InMail. The administrators requested for excerpts detailing the objectives of the study and some of the questions that may be asked. After a consideration of the questions and the objectives, the researcher 
was granted permission to carry on with the questions based on an agreement not to disclose member's pictures and names on publishable material. However, this was not the case in the ST group as the administrator informed the researcher that the questions being posed were out of sync with the interests of the group's membership. The consent obtained from the VSA was expected as it was a closed group with membership only open to persons with interests in viable systems theory, viable systems models and the real-world application of both theory and model.

The researcher created a separate platform within the wider platform with a note informing members of the study that was being carried out and the reason why their opinion was being sought. Individuals who were interested in the study were asked to indicate their interest by liking the introductory statement posed by the researcher. In total, 46 likes were received over a three-day duration. At the time of the study, VSA had membership of 431 individuals hence representing an estimated $10 \%$ of the total membership. The researcher, serving as a facilitator introduced new threads to deal with different questions which were aimed at achieving the study's objective. The exercise lasted for a duration of approximately seven (7) months, between July 2012 and February 2013. However, over this period, only 32 discussants participated in the various threads with active participation recorded for 17 discussants. Active participation refers to discussants who contributed more than once across the six discussion threads established over by the researcher. To the researcher, this was an indication of the acceptability of the study and the protocol utilized in eliciting data from the discussants. Table 1 details the number of threads, the number of posts on each thread and the number of discussants and active discussants who contributed to each thread.

Table 1. Discussion Threads, Posts and Discussants

\begin{tabular}{|l|l|l|l|}
\hline Thread & Number of Posts & Summary of Discussants & Number of Active Discussants \\
\hline 1 & 49 & 34 & 17 \\
\hline 2 & 72 & 21 & 17 \\
\hline 3 & 38 & 18 & 17 \\
\hline 4 & 23 & 17 & 17 \\
\hline 5 & 85 & 19 & 17 \\
\hline 6 & 27 & 17 & 17 \\
\hline 6 & 294 & 126 & 17 \\
\hline
\end{tabular}

Source: Author's fieldwork (2014)

From Table 1, the intensity of the discussions on the AODF can be deciphered accordingly. The number of posts mentioned exclude the posts made by the researcher during facilitation. Table 2 provides the demographics of the active discussants.

Table 2. Demographics of Discussants

\begin{tabular}{|l|l|l|l|l|}
\hline Code & Position & Expertise & $\begin{array}{l}\text { Relevant experience } \\
\text { (years })\end{array}$ & Location \\
\hline A & Professor & Sustainable Business Models & 22 & United Kingdom \\
\hline B & Associate Professor & Construction Informatics & 19 & Oman \\
\hline C & Professor & Systems thinking application in Business & 30 & Switzerland \\
\hline D & Research Fellow & Systems thinking & Switzerland \\
\hline E & Senior Project Manager & Systems thinking application & 12 & United Kingdom \\
\hline G & Management Consultant & $\begin{array}{l}\text { Target Operating Models development } \\
\text { using VSM }\end{array}$ & 25 & Germany \\
\hline
\end{tabular}




\begin{tabular}{|c|c|c|c|c|}
\hline $\mathrm{H}$ & Software Expert & Systems thinking and Cybernetics & 28 & United Kingdom \\
\hline I & Chief Solutions Architect & Cybernetics and Change Management & 28 & Canada \\
\hline $\mathrm{J}$ & Solutions Manager & Systems Integration & 19 & Switzerland \\
\hline $\mathrm{K}$ & Professional Consultant & $\begin{array}{l}\text { Leadership and system design and } \\
\text { modelling }\end{array}$ & 14 & Italy \\
\hline $\mathrm{L}$ & Business Solutions Expert & $\begin{array}{l}\text { Systems dynamics and Next generation } \\
\text { Enterprise }\end{array}$ & 14 & Italy \\
\hline $\mathrm{M}$ & Management Consultant & Organizational analysis and design & 18 & South Africa \\
\hline $\mathrm{N}$ & Management Cybernetician & Management Cybernetics & 32 & United States of America \\
\hline $\mathrm{O}$ & Financial/ economic Analyst & International/policy development & 23 & Netherlands \\
\hline $\mathrm{P}$ & Researcher & Systems thing in Construction & 18 & Hong Kong \\
\hline
\end{tabular}

Source: Awuzie (2014)

The researcher obtained rich data from the postings made by these discussants. A look at the experience of the discussants- an estimated average of 19.5 years- indicates that the researcher was able to obtain relevant viewpoints on the suitability and applicability of the VSM and its underlying theory in carrying out organizational diagnosis of the IDS. However, it is beyond the scope of this study to discuss the views elucidated in those posts. As such, the study will concern itself with reflecting on the usefulness or otherwise of the AODF in the elicitation of data from systems thinking experts across the world.

\subsection{Reflections on the Asynchronous Online Discussion Forum's Utility}

The AODF and other online discussion forums have been presented as veritable platforms for knowledge creation and exchange across targeted groups or individuals in geographically dispersed locations in a cost-effective manner. Yet, of interest to this study, is its use in facilitating data collection for qualitative research. In subsequent sections, the researcher will reflect on the aspects of the ADOF, highlighting its utility and identifying challenges associated with the approach.

\section{Merits}

During the study, the researcher identified the following advantages of the ADOF;

\section{Ease of transcription}

The ADOF platform allowed for the automatic generation of transcripts from discussant postings. This was a significant advantage when the labourious and expensive nature of transcribing interviews is considered. This advantage happens to be the most common advantage as it has been mentioned in similar studies ${ }^{[8,17,13]}$. The researcher was able to generate the entire transcripts from the LinkedIn group platform.

\section{Control of discussion patterns}

The facilitator's ability to control the tone and direction of discussions between discussants during conventional focus group interview sessions remains a salient challenge. The facilitator is expected to manage the power-relations and dynamics which are usually present even within homogenous groups during the discussions. Such dynamics usually affect the validity of the views expressed therein as certain discussants are afraid not to upset their colleagues or organization even when confidentiality and anonymity has been guaranteed by the facilitator. The purposive recruitment of highly homogenous groups has been referred to as a panacea for resolving this challenge, but the efficacy 
of such selection process remains imagined. But in the ADOF study described, the facilitator did not notice any situation which highlighted the incidence of such scenarios from the posts.

\section{Coverage of wide geographical area}

The coverage area of the discussant cohort can be deciphered from Table 2. This is another salient advantage of the ADOF. It brought together professionals who were involved with the Viable Systems Model thus providing a platform for multi-, inter-, and transdisciplinary co-creation of knowledge on the applicability and suitability of the VSM for diagnosing TMOs. Hence enabling co-creation of knowledge: one of which the identification a set of pre-set themes which the researcher used in outlining the shortcomings of the IDS that was being diagnosed in the wider study.

\section{Convenience}

Based on its asynchronicity, the platform proved to be convenient for the researcher and the community of experts as they were only obliged to post comments at their convenience. Also, this enabled them to engage in thoughtful reflections before responding to previous posts on the threads. It prevented spontaneity among the discussants as would have been the case if the event of focus group discussions or synchronous online discussion forums. The researcher was notified concerning new posts through an alert as enabled by the LinkedIn, hence not needing to remain online in wait of new posts.

\section{Relevance of contributions}

Because of the purposive nature of discussant recruitment and the willingness of individuals to partake in the study without coercion, the discussions were found to be relevant to the theme of the study. This observation contributed to a more than 50\% retention rate in the discussant cohort as shown in Table 1. This mere fact serves as a morale booster for the researcher as it reinforces the salience of the study being undertaken to the world of practitioners, beyond the academic disciplinary boundaries but also stretching into the real-world context. The number of discussants was not considered an anomaly as conventional focus groups will usually consist of between 7 and 10 discussants whereas in this case, 17 active discussants were identified from the threads.

\section{Challenges}

The AODF as deployed in this study did not come without challenges. One of such challenges is discussed below.

\section{Sustaining discussions on threads}

The use of the AODF was not a smooth on after all as the researcher encountered difficulties with sustaining the tempo on threads. At some points the threads appeared dormant despite prompts from the researcher to encourage further contributions. Also, in some instances the researcher had to refer to sister threads on the same matter or some other discussant's opinion to see if that will lead to renewed interest. Whereas this worked in some instances, it failed to do so in other instances. Judging from contents of relevant literature, this is a common issue with AODFs ${ }^{[15,10]}$. Perhaps this may be because of the ling time frame for data elicitation. However, the researcher felt constrained in deciding on when to stop seeking for new contributions as saturation was not reached on two of the questions that were posted on the platform. Eventually, the choice of the appropriate time to stop posing questions was influenced by the timelines of the wider study. Yet, two years after, comments were still being posted on the platform, obviously due to its asynchronicity, until the researcher sought the permission of the administrator to close the threads.

\section{Conclusion}

The recruitment of interviewees/discussants to participate in qualitative BE research projects has been identified as a challenge to most $\mathrm{BE}$ researchers. Besides this, the labourious and expensive nature or transcribing qualitative data posed another challenge to these cohort of researchers. As a result, these cohort seem to overtly rely on the quantitative methods of data collection. This is evident in the prevalence of quantitative research projects within the BE domain.

Aware of these challenges, this study set out to provide an exposé into the utility of IMC techniques such as AODF in surmounting these challenges. It relies on a study conducted between 2013 and 2014 within which the AODF was deployed towards accomplishing certain aspects of the study. Accordingly, a highlight of the advantages accruing from the utility of the approach and the challenges, was provided herein by the researcher. From the evidence presented in 
the paper, it is evident that the benefits far outweigh the disadvantages. However, it must be noted that the AODF's utility is predicated on the level of knowledge of probable discussants on the usage of the internet as well as access. In furtherance to this, it should be noted that the cost-effectiveness of the approach as used in this study, resulted from the use of an already established platform- LinkedIn. It is believed that this will be different if the researcher must develop a bespoke platform for his study.

It is expected that this study will contribute in generating interest around the use of IMC techniques such as the AODF for qualitative data collection purposes within the realm of BE research.

\section{References}

[1] Abawajy, J. (2012) Analysis of asynchronous online discussion forums for collaborative learning, International Journal of Education and Learning, Vol. 1 (2) pp. 11-21

[2] Andresen, M. A. (2009). Asynchronous discussion forums: success factors, outcomes, assessments, and limitations. Educational Technology \& Society, Vol. 12 (1), 249-257

[3] Awuzie, B.O (2014) A viable infrastructure delivery systems model for achieving socio-economic benefits in the Nigerian Oil and Gas Industry Unpublished $\mathrm{PhD}$ thesis University of Salford

[4] Beer, S. (1984). The viable system model: its provenance, development, methodology and pathology. Journal of the operational research society, Vol. 35 (1) pp. 7-25

[5] Brownlow, C. and O'Dell, L. (2002) Ethical issues for qualitative research in On-line communities, Disability and Society, Vol17 (6) pp. 685694

[6] Bryman, A. (2012) Social Research Methods 4th edition. Oxford. Oxford University Press

[7] Chan, J.C.C, Hew, K.F, and Cheung, W.S. (2009) Asynchronous online discussion thread development: examining patterns and peer-facilitation techniques. Journal of Computer Assisted Learning Vol. 25(5) pp. 438-452

[8] Dringus, L. P., and Ellis, T. (2005) Using data mining as a strategy for assessing asynchronous discussion forums Computers and Education Vol.45 (2005) pp.141-160

[9] Eisenhardt, K. M. and Graebner, M. E. (2007). Theory Building from Cases: opportunities and challenges. Academy of Management Journal Vol.50 (1) pp. 25-32

[10] Fu, E.L.F, Van Aalst, J., and Chan, C.K.K (2016) Toward a classification of discourse patterns in asynchronous online discussions International Journal of Computer-Supported Collaborative Learning Vol. 11(4) pp. 441-478

[11] Gao, F., Zhang, T., and Franklin, T. (2013) Designing asynchronous online discussion environments: recent progress and possible future directions. British Journal of Educational Technology Vol. 44(3) pp.469-483

[12] Gilbert, P.K., and Dabbagh, N. (2005) How to structure online discussions for meaningful discourse: a case study British Journal of Educational Technology Vol 36 (1) pp.5-18

[13] Hew, K.F., and Cheung, W.S. (2011) Higher-level knowledge construction in asynchronous online discussions: an analysis of group size, duration of online discussion, and student facilitation techniques Instructional Science Vol. 39 (3) pp.303-319

[14] Hoverstadt, P. (2008). The Fractal Organization: Creating sustainable organizations with the viable system model. West Sussex, John Wiley and Sons Ltd.

[15] Hung, M., and Chou, C. (2014) The Development, Validity, and Reliability of Communication Satisfaction in an Online Asynchronous Discussion Scale Asia-Pacific Educational Research Vol. 23(2) pp.165-177

[16] Im, E.-O. \& Chee, W. (2006). An online forum as a qualitative research method: Practical issues. Nursing Research, 55, 267.

[17] Lim, C.P., and Tan, S.C., (2001) Online discussion boards for focus group interviews: an exploratory study. Journal of Educational Enquiry Vol.2 (1) pp.50-60 
[18] Maestre, J.F., MacLeod, H., Connelly, C.L., Dunbar, J.C. Beck, J., Siek, K.A., and Shih, P.C. (2018) Defining through expansion: conducting asynchronous remote communities (ARC) research with stigmatized groups Proceedings of the 2018 CHI Conference on Human Factors in Computing Systems, Montreal Canada, 21st -26th April 2018.

[19] McKiernan, A., Ryan, P., McMahon, E. and Butler, E. (2017) Qualitative analysis of interactions on an online discussion forum for young people with experience of romantic relationship breakup Cyberpsychology, Behavior, And Social Networking Vol 20 (2) pp. 78-82

[20] Moore T., McKee, K, McLoughlin, P. (2015) Online focus groups and qualitative research in the social sciences: their merits and limitations in a study of housing and youth People, Place and Policy Vol. 9 (1) pp. 17-28.

[21] Nandi, D., Hamilton, M., and Harland, J. (2012) Evaluating the quality of interaction in asynchronous discussion forums in fully online courses, Distance Education, Vol 33(1) pp. 5-30

[22] Plano Clark, V.L and Creswell, J.W (2015) Understanding Research: a consumers' guide. 2nd edition. Boston. Pearson Education

[23] Sydow, J. and Braun, T. (2018) Projects as temporary organizations: An agenda for further theorizing the interorganizational dimension International Journal of Project Management Vol. 36 (1) pp.4-11

[24] Ratislavová, K., and Ratislav, J., (2014) Asynchronous email interview as a qualitative research method in the humanities Human Affairs Vol. 24 (4) pp. $452-460$

[25] Redlich-Amirav, D., and Higginbottom, G. (2014). New Emerging Technologies in Qualitative Research. The Qualitative Report, Vol.19(26), pp. 1-14.

[26] Rezabek, R.J., (2000) Online Focus Groups: Electronic Discussions for Research Qualitative Social Research, Vol 1 (1) Art. 18

[27] Turney, L and, Pocknee, C, (2005) Virtual focus groups: new frontiers in research International Journal of Qualitative methods Vol. 4(2) pp. $32-43$ 